\title{
Dust destruction in the ISM: a re-evaluation of dust lifetimes
}

\author{
A. P. Jones ${ }^{1}$ and J. A. Nuth $\mathrm{III}^{2}$ \\ ${ }^{1}$ Institut d'Astrophysique Spatiale (IAS), Bâtiment 121, Université Paris 11 and CNRS, 91405 Orsay, France \\ e-mail: Anthony.Jones@ias.u-psud.fr \\ 2 Astrochemistry Laboratory - Solar System Exploration Division, NASA/GSFC, Code 691, Greenbelt, MD 20771, USA \\ e-mail: Joseph.A.Nuth@nasa.gov
}

Received 16 March 2010 / Accepted 10 April 2011

\begin{abstract}
Context. There is a long-standing conundrum in interstellar dust studies relating to the discrepancy between the time-scales for dust formation from evolved stars and the apparently more rapid destruction in supernova-generated shock waves.

Aims. We re-examine some of the key issues relating to dust evolution and processing in the interstellar medium.

Methods. We use recent and new constraints from observations, experiments, modelling and theory to re-evaluate dust formation in the interstellar medium (ISM).

Results. We find that the discrepancy between the dust formation and destruction time-scales may not be as significant as has previously been assumed because of the very large uncertainties involved.

Conclusions. The derived silicate dust lifetime could be compatible with its injection time-scale, given the inherent uncertainties in the dust lifetime calculation. The apparent need to re-form significant quantities of silicate dust in the tenuous interstellar medium may therefore not be a strong requirement. Carbonaceous matter, on the other hand, appears to be rapidly recycled in the ISM and, in contrast to silicates, there are viable mechanisms for its re-formation in the ISM.
\end{abstract}

Key words. ISM: abundances - dust, extinction

\section{Introduction}

Two major and separate populations of grains arise naturally from the circumstellar outflows of carbon-rich and oxygen-rich stars. These populations are carbonaceous materials, including amorphous (hydrogenated) carbons, graphite, diamond and metal carbides, and oxides consisting primarily of amorphous silicates. Until about 1980 the common assumption had always been that interstellar grain populations had their roots in stellar outflows (Mathis et al. 1977) with only minor modifications arising in certain regions of the interstellar medium (ISM), for example, the growth of volatile ice mantles or the formation of carbonaceous residues on the grains (Greenberg 1983; Schutte 1996; Sandford et al. 1997).

The observation of shock-induced dust destruction (Routly \& Spitzer 1952; Cowie 1978; Seab \& Shull 1983; Welty et al. 2002) introduces a severe modification of the dust population: the complete destruction of all grains in the ISM on a timescale $\sim 10^{8} \mathrm{yr}$ (Jones et al. 1994, 1996). If this rapid destruction does occur, then the vast majority of grains found in the ISM must be (re-)formed there. However, the models that predict rapid grain destruction in the ISM often do not consider in detail the problems associated with forming refractory grains from diffuse vapors at the relatively low temperatures and pressures of the ISM. These authors clearly recognised that their calculations predicted "unreasonably" high dust destruction rates, given that there is no apparent way to remake the grains in the ISM, but were unable to resolve this conundrum. Thus, the consequential need to re-form separate populations of carbon-rich and oxygen-rich grains - post erosion - in the low-density ISM has not yet been satisfactorily addressed.
In what follows we consider the problems of grain formation and destruction in a global context and try to constrain the nature of the dust formed in the ISM following erosion in strong shocks. We give an overall evaluation of the condition of solids in the ISM, including an assessment of where most of the material found there might have formed as well as an estimate of the fraction of the material that has been destroyed in shocks and recondensed in the interstellar medium. We point out potential flaws in the usual calculations of the lifetime of dust in the galaxy and suggest an approach that might help to resolve this problem.

\section{Dust in the ISM}

The mineralogy of grains in the ISM varies, depending on the particular phase, e.g., the mineralogy of the dust in molecular cloud cores differs from that of the diffuse ISM, although the same refractory phases may be common to both. In Table 1 we give our best estimates of the abundances of the ISM dust constituents as a fraction of the dust mass in the broad groups: carbonaceous $(\mathrm{C})$, silicates/oxides $(\mathrm{O})$ and volatile ices $(\mathrm{V})$. The volatiles such as $\mathrm{H}_{2} \mathrm{O}$ only enter into the solid state in cold, molecular clouds. Here we have counted the residues of warmed, irradiated ices as a part of the amorphous carbon component. The ordering of the constituents in Table 1 reflects the degree of certainty in the existence of the individual components in the ISM with a second level of ordering based on abundance. The first eight constituents in Table 1 have been identified in meteorites. Amorphous carbon grain coatings have not been isolated from meteorite matrices directly but are inferred from the presence of highly-anomalous, isotopic abundances of $\mathrm{N}, \mathrm{C}$ 
Table 1. The mineralogy of interstellar grains.

\begin{tabular}{lcl}
\hline \hline Component & Fraction of solid phase & Primary spectral features \\
\hline Amorphous carbons $\left(\mathrm{sp}^{2}, \mathrm{sp}^{3}\right), \mathrm{a}-\mathrm{C}: \mathrm{H}, \mathrm{PAHs}$ & $>80 \% \mathrm{C}$ & aromatic emission bands, $\approx 200 \mathrm{~nm}$ features, $3.4 \mu \mathrm{m} \mathrm{C}-\mathrm{H}$ stretch \\
Nanodiamonds $\left(\mathrm{sp}^{3}\right.$ carbon) & $\approx 10 \% \mathrm{C}$ & tertiary C-H stretch $(2.9 \mu \mathrm{m})$, Far UV absorption, $22 \mu \mathrm{m}$ peak \\
$\mathrm{Graphitic}$ carbon $\left(\mathrm{sp}^{2}\right)$ & $\approx 5 \% \mathrm{C}$ & $217.5 \mathrm{~nm}$ peak \\
$\mathrm{SiC}$ & trace C & Broad $11.2 \mu \mathrm{m}$ peak \\
\hline Amorphous silicate $(\mathrm{Fe}, \mathrm{Mg})_{x} \mathrm{SiO}_{y}$ & $>95 \% \mathrm{O}$ & 9.7 and $18 \mu \mathrm{m}$ peaks \\
Corundum $\left(\mathrm{Al}_{2} \mathrm{O}_{3}\right)$ & trace $\mathrm{O}$ & $12-13 \mu \mathrm{m}$ peak \\
Spinel $(\mathrm{Fe}, \mathrm{Mg}) \mathrm{Al}_{2} \mathrm{O}_{4}$ & trace $\mathrm{O}$ & $12-13 \mu \mathrm{m}$ peak \\
Crystalline silicates: amorphised in the ISM & trace O & $11.3 \mu$ m olivine sub-peak to the $10 \mu \mathrm{m}$ feature, many FIR features \\
\hline Water ice polar $($ plus organics) & $>90 \% \mathrm{~V}$ & $3,6,12,45,60 \mu \mathrm{m}$ peaks \\
Non-polar ices $\left(\mathrm{N}_{2}, \mathrm{O}_{2}\right)$ & $\approx 5 \% \mathrm{~V}$ & indirect effects on dissolved species \\
Non-Polar ices $\left(\mathrm{CO}, \mathrm{CO}_{2}\right)$ & $\approx 5 \%$ & $4.67(\mathrm{CO}), 4.27 \& 15.2\left(\mathrm{CO}_{2}\right) \mu \mathrm{m}$ peaks \\
\hline MgS, CaS, $\mathrm{SiS}_{2}:$ react with $\mathrm{H}_{2} \mathrm{O}$ in the ISM & $?$ & 28,40 and $22 \mu \mathrm{m}$ peaks $($ respectively) in CS shells \\
\hline
\end{tabular}

Notes. Solid phases: $\mathrm{C}=$ solid carbonaceous grains, $\mathrm{O}=$ oxide grains, $\mathrm{V}=$ volatile ices .

and D found in the matrix (Zinner 1988) and in IDPs (e.g., Zinner 1988; Messenger \& Walker 1996), which are indicative of their possible formation via ion-molecule reactions or surface-mediated processes in cold cloud cores. The presence of pre-solar nanodiamond, graphite (sometimes better described as poorly graphitized carbon) and $\mathrm{SiC}$ in the meteorite matrix is clear (Meyer \& Zinner 2006; Bernatowicz et al. 2005). Note that the primary spectral features listed in Table 1 may not be directly attributable to only one particular component; e.g., the infrared emission bands that have been attributed to PAHs (Leger \& Puget 1984; Cohen et al. 1986), to various forms of amorphous carbon such as hydrogenated amorphous carbon (HAC or a-C:H: Duley 1985; Pino et al. 2008) or quenched carbonaceous composite (QCCs: Sakata et al. 1983) materials which can be regarded as aggregated PAHs linked by aliphatic hydrocarbons (e.g., Jones 1990). Clearly, for such materials to explain the IR emission bands their sizes would have to be comparable to those invoked for PAHs or very small carbon grains (e.g., see Jones \& d'Hendecourt 2000, for a calculation of typical nanodiamond temperatures in the ISM). These IR emission features could also arise from the amorphous, carbonaceous outer layers on nanodiamonds (Jones \& d'Hendecourt 2000), though measurements of the IR spectra of chemically processed, meteoritic diamonds do not resemble the above-mentioned bands (Mutschke etal 1995; Sheu et al. 2002; Jones et al. 2004).

\section{Dust formation}

Stardust, literally the dust formed around stars, is formed at the end of the life of lower mass stars as they transit to the red giant branch (RGB), asymptotic giant branch (AGB) and planetary nebula (PN) phases and as more massive stars explode as SNe. Presolar grains originating from these sources are clearly indicated (e.g., Meyer \& Zinner 2006). Although the contribution from SNe could be dominant, we do not yet have clear observational evidence that $\mathrm{SN}$ ejecta directly form significant quantities of dust. Observations of dust formation in SNe seem to indicate an average dust production per core collapse SN of not more than $0.01 M_{\odot}$ and little or no evidence for dust formation around type Ia $\mathrm{SNe}$. Thus, $\mathrm{SNe}$ can seemingly only explain a very small fraction of stardust.

\subsection{In circumstellar media}

Dying stars are the best natural laboratories for the study of the grain formation process, especially the formation of oxide/silicate grains. Mass outflows from oxygen-rich AGB and RGB stars are roughly steady state, homogeneous, dynamic atmospheric escape processes (Kozasa \& Hasegawa 1987; Fleischer et al. 1991, 1992, 1995; Jeong et al. 2003). The stellar atmosphere initially contains only vapor at temperatures well in excess of $2000 \mathrm{~K}$ : gas expansion away from the stellar surface leads to smooth decreases in both the temperature and pressure of the gas. This cooling eventually leads to the nucleation of refractory grains. These grains then serve as condensation nuclei that rapidly deplete the gas of its remaining, less refractory vapors. Nucleation and grain growth occur on rapid timescales and produce highly amorphous, chemically heterogeneous grains that become more ordered as they are annealed in the outflow (Nuth \& Hecht 1990; Nuth et al. 1996, 2002). As an example, it is inferred that Fe- and Mg-silicates condense out as separate grain populations; iron silicates require much higher temperatures to anneal than do magnesium silicates. Therefore, for high mass outflow rates, where the condensation temperatures are higher due to higher concentrations of the condensable elements, some magnesium silicates can anneal to crystallinity while the iron silicates remain amorphous, explaining the observed "pure" magnesium silicates and the absence of evidence for iron-bearing silicate minerals in these sources (Nuth et al. 2000, 2002). However, it is possible that residual carbon in the gas could reduce the $\mathrm{Fe}$ to metallic iron during the condensation process (Davoisne et al. 2006), a scenario that is also consistent with observations.

Grain formation around carbon-rich AGB stars is more complex than for oxygen-rich stars due to the great richness of the carbonaceous phase and to the greater thermal stability of species such as metallic carbides and poorly graphitized carbon. These factors can lead to the formation and growth of grains in the pre-expansion atmospheres of carbon-rich AGB stars. Bernatowicz \& Cowsik (1997) have shown that the large, presolar graphite grains isolated from meteorites grew under equilibrium conditions for time periods of at least a year around thermodynamically stable nuclei of titanium carbide prior to their expulsion from the star. The growth of the largest grains may have required periods as long as $10^{5} \mathrm{yr}$ (Michael et al. 2003). Even though other carbonaceous grains might nucleate homogeneously in these carbon-rich outflows, the presence of pre-existing condensation nuclei and large graphite and $\mathrm{SiC}$ grains that might act to heterogeneously accrete amorphous carbon vapor make it more difficult to construct a self-consistent model of grain formation in such environments.

The interesting case of rapid carbonaceous and silicate dust formation in some novae (e.g., Shore \& Gehrz 2004; 
Evans et al. 2005) may perhaps shed some light on the possibility of rapid dust formation in the ISM. In these objects dust appears to form very efficiently and very fast once nucleation has occurred. It has been suggested that ionisation may actually play a key role in this rapid appearance of dust (Shore \& Gehrz 2004). Additionally, novæ seem to be able to perform the simultaneous nucleation of carbonaceous and silicate grains, (e.g., Evans et al. 2005). Although, it is not clear whether this is due to coeval multi-phase dust formation or due to chemically-distinct and spatially and/or temporally separated mass-loss episodes. Given that the gas in the shells around novæ, where dust formation occurs, are extremely dense it seems unlikely that similar dust formation processes could occur in the ISM.

Clearly evolved carbon stars are important sources of carbon-rich dust. There is now increasing observational evidence that this dust primarily consists of hydrogenated amorphous carbonaceous solids that form and evolve in their atmospheres and extended shells (e.g., Goto et al. 2003) and after their injection into in the ISM (e.g., Jones 1990; Jones et al. 1990; Dartois et al. 2004; Pino et al. 2008). Hydrogen implantation into grains, in conjunction with photo-processing in energetic regions of the ISM, e.g., HII regions, photon-dominated regions and shock waves, will probably pollute any pure carbon phase and lead to significant changes in its chemistry and structure.

Chiar et al. (1998) observed the 3.4 micron C-H stretch in the outflow around the proto-planetary nebula CRL 618, thus showing that the band carrier can form in the ejecta from evolved carbon-rich stars. Carbonaceous grains might also form in supernovae (Clayton et al. 1999).

The dust formation process may be aided by effects of stellar thermal pulsations, which imply that the dust formation and ejection may be episodic, as implied by the observed concentric dust shells around evolved stars such as IRC+10216 (Mauron \& Huggins 1999).

\subsection{In the interstellar medium}

Laboratory analogues of interstellar carbon dust (e.g., Dartois et al. 2004) show that hydrogen-rich (>50 atomic $\% \mathrm{H}$ ) amorphous hydrocarbon solids can explain the observed interstellar absorption bands at 3.4, 6.85 and 7.25 $\mu \mathrm{m}$. Dartois et al. (2004) also show that the thermal annealing of this material is accompanied by an increase in the aromatic component, i.e., an "aromatisation". Such a transformation and evolution of interstellar hydrocarbons was proposed by Duley et al. (1989); Jones (1990); Jones et al. (1990) as the result of photon-driven processing of hydrogen-rich hydrocarbon mantles accreted onto silicate grains in the ISM. However, these hydrocarbon materials could also be of stardust origin. A progressive loss of hydrogen, and associated "aromatisation", of hydrocarbon grains would lead to a smaller band gap material and an increase in the aromatic content. The end point of this process is probably a low-density, aromaticrich material that could dissociate into its constituent aromatic molecular components (PAH-like species), and the aliphatic and olefininc hydrocarbon bridging structures (e.g., Duley 2000; Petrie et al. 2003; Pety et al. 2005; Jones 2005, 2010) as a result of UV photo-processing and/or fragmentation in grain-grain collisions (Jones 2005; Jones et al. 1996).

A $3.4 \mu \mathrm{m}$ absorption feature appears along several lines of sight in the diffuse interstellar medium (Butchart et al. 1986; Adamson et al. 1990; Sandford et al. 1991; Pendleton et al. 1994) but is not observed in the spectra of dust in dense molecular clouds, though this could simply mean that it becomes obscured by the deposition of an ice mantle or by chemical effects
(Mennella 1998). Because it can be formed via UV processing of organic ice mantles (e.g., Greenberg 1989; Greenberg et al. 1995; Bernstein et al. 1999, 2001, 2002; Dworkin et al. 2001; Hudson \& Moore 2004) its presence in the diffuse interstellar medium is then often cited as a measure of the importance of such processes.

However, spectropolarimetric observations of the interstellar $3.4 \mu \mathrm{m}$ absorption feature towards the Galactic Centre (Adamson et al. 1999; Chiar et al. 2006) and in the Seyfert 2 galaxy NGC 1068 (Mason et al. 2007) show levels of polarisation below that predicted by carbonaceous mantle-silicate core dust models. This strongly indicates that the $3.4 \mu \mathrm{m}$ band originates from a population of small, non-polarising, carbonaceous grains that share characteristics with the $217.5 \mathrm{~nm}$ carrier but that are distinct from the silicate grain population responsible for the polarisation of the $9.7 \mu \mathrm{m}$ amorphous silicate $\mathrm{Si}-\mathrm{O}$ stretching band (Adamson et al. 1999; Chiar et al. 2006; Mason et al. 2007). This spectropolarimetric observational constraint would be consistent with the rapid, UV photon-driven dehydrogenation of carbonaceous dust in the low density ISM. It could also, in principle, be explained by a process that inhibits or preferentially removes carbonaceous species from the surfaces of silicate grains, however, and as far as we are aware, no such viable process has yet been found (see Sect. 4.2.)

It is easy to imagine that small, stochasticaly-heated carbonaceous grains could dehydrogenate, as a result of the direct UV photo-dissociation of $\mathrm{C}-\mathrm{H}$ bonds or as an effective cooling mechanism, and that they could also react with ambient hydrogen to reform $\mathrm{C}-\mathrm{H}$ bonds on their surfaces, achieving an equilibrium composition that depends on the local conditions. Sorrell (1989), Sorrell (1990) and Hecht (1991) proposed that grains entering the diffuse interstellar medium lost most of their hydrogen to form graphitic dust, thus increasing the strength of the $217.5 \mathrm{~nm}$ feature and, upon entering denser media, the grains react with hydrogen, which disrupts the aromatic/graphitic structures and weakens the $217.5 \mathrm{~nm}$ band (Sorrell 1991). However, this appears to be incompatible with the above-mentioned lack of a $3.4 \mu \mathrm{m}$ absorption feature in dense molecular clouds.

If refractory atoms and molecules slowly accrete onto the surfaces of surviving grains only after they have reached the comparative safety of a molecular cloud (rather than in the diffuse ISM), then such atoms may have the opportunity to react with additional adsorbed atomic and molecular species before being buried beneath the growing grain surface. The most abundant atomic species is $\mathrm{H}$ and the most abundant molecules are $\mathrm{H}_{2}, \mathrm{CO}$ and $\mathrm{H}_{2} \mathrm{O}$. If these reactions occurred, the $\mathrm{SiO}$, and $\mathrm{Fe}$ and $\mathrm{Mg}$ atoms generated by the erosion of grains in supernova shocks might never reform the initial silicate compounds observed in the ISM, but might instead form a complex ice. Experiments made with mixtures of silane $\left(\mathrm{SiH}_{4}\right)$, iron pentacarbonyl $\left(\mathrm{Fe}(\mathrm{CO})_{5}\right)$ and $\mathrm{H}_{2} \mathrm{O}$ ice condensed at very low temperatures $(\sim 10 \mathrm{~K})$ have demonstrated that a silicate can form from such a mixture, especially if the mixture were irradiated with cosmic-ray energy protons prior to warm-up so as to form chemical radicals in the ice (Nuth \& Moore 1989). However, a major problem with the results of this study is that the IR spectrum of the warmed silicate residue does not resemble typical IR spectra observed for silicates in the ISM. Based on previous laboratory work, Hallenbeck et al. (1998, 2000) showed that the thermal annealing $(T \geq 1000 \mathrm{~K})$ of a highly amorphous silicate condensate resulted in more ordered material with an IR spectrum displaying narrower peaks and absorption features that more closely resemble typical ISM silicate spectra. However, no natural process has been identified that would heat grains to temperatures 
significantly in excess of $1000 \mathrm{~K}$ in the ISM for sufficiently long time periods to reproduce the observed silicate spectral profile. This process would have to occur on a considerably shorter timescale than grain destruction in order to transform the recondensed refractory material into the observed amorphous interstellar silicate.

Thus, any reasonable scenario for grain formation in the ISM will yield materials whose spectral properties do not match observations (Nuth et al. 1998). We suggest that the mineralogy of the majority of interstellar silicate grains is established in oxygen-rich circumstellar outflows and is only slightly modified by subsequent interstellar processes. However, the processing and recycling of carbonaceous matter may be rather fast, and more complex, in comparison.

Recently Jenkins (2009), in a detailed study of ISM elemental depletions, came to an interesting conclusion about interstellar oxygen. Its seems from this work that oxygen is incorporated into a solid form at a rate that appears to be well in excess of that required for silicate and oxide grain formation. In a follow-up study Whittet (2009) postulated that this excess depleted oxygen could be in the form of O-bearing C-rich matter similar to an "organic refractory" material.

\section{Dust processing in the ISM}

The primary dust destruction processes that lead to the erosion of the grain constituent atoms into the gas are sputtering in gasgrain collisions and vaporisation in grain-grain collisions in supernova shock waves. In general, vaporisation is subordinate to sputtering and to the shattering that occurs, along with vaporisation, in grain-grain collisions (Jones et al. 1996). Of the destructive processes it is therefore sputtering in gas-grain collisions that primarily determines the lifetime of dust in the ISM and is an important destructive process for all dust grains in interstellar, supernova-generated shock waves and also stellar jets and outflows (e.g., Guillet et al. 2007, 2009).

The atom/ion sputtering yield is less than unity and so the process is accompanied by implantation into the grain. Thus, atom/ion implantation could actually lead to a net increase in the grain mass and the "destruction" process could result in grain "growth" (e.g., Gray \& Edmunds 2004). However, the implantation process leads to major structural changes in the grains (e.g., the amorphisation of crystalline grains), and to the introduction of porosity, if the incident atom/ion energies are sufficiently high, i.e., 4-50 keV (e.g., Demyk et al. 2001)

At very high incident energies, i.e., of the order of $\mathrm{MeV}$ per nucleon, the incident ions can completely pass through a grain and leave it unchanged. Day (1977) showed that $\mathrm{MeV}$ proton irradiation (1 MeV per nucleon) has no significant effect upon the IR signatures of forsterite or enstatite grains. However, Krätschmer \& Huffman (1979) showed that $1.5 \mathrm{MeV}$ $\mathrm{Ne}$ ions (75 keV per nucleon) can disorder crystalline forsterite and substantially alter its optical properties.

Crystalline silicates present an interesting problem and demonstrate a very significant difference between the properties of grains in the ISM versus the crystalline silicates observed to form in outflows of evolved stars (e.g., Waters et al. 1996) and young main-sequence stars (e.g., Waelkens et al. 1996). Given this, there should be some fraction of the interstellar silicate grain population that is crystalline, yet no evidence for crystalline silicates (Dorschner et al. 1995; Henning et al. 1995; Henning \& Mutschke 1997) has been found in the general ISM. There is the possibility that as much as $5 \%$ of interstellar silicates could be crystalline, but that these crystalline features would lie below observational detection limits (Li \& Draine 2001). Based on the structure of the $10 \mu \mathrm{m}$ silicate feature measured along a single line of sight towards the galactic center, Kemper et al. (2004) calculated an upper limit on the fraction of crystalline silicates along that very long path to be $2.4 \pm 2.4 \%$.

If interstellar grains are destroyed on rapid timescales in the ISM then the lack of crystalline silicate grains is perfectly understandable. Since these silicates only form in a small fraction of the oxygen-rich stellar outflows, crystalline grains would be relatively rare to begin with. Rapid grain destruction coupled with no feasible mechanism by which crystalline silicates can form or anneal would predict a dearth of crystalline silicates in the ISM and in the presolar grain population. However, given the survival of a host of somewhat delicate carbonaceous materials in the ISM, the new dilemma either becomes explaining the preferential destruction of crystalline interstellar silicates versus carbonaceous grains or finding an alternative explanation for the observational data. One good alternative is the deterioration of crystalline silicates upon long exposure to cosmic radiation (e.g., Day 1974; Jäger et al. 2003; Bringa et al. 2007)

However, since some crystalline silicate grains are observed in meteorites and IDPs that are demonstrably presolar (Messenger et al. 2003; Keller \& Messenger 2004; Nguyen \& Zinner 2004), some small fraction of silicates in the ISM must be crystalline.

Supernovae shock waves destroy refractory grains: this is an observational fact (Seab \& Shull 1983). However, establishing the lifetimes of grains in the ISM requires the use of models that contain assumptions ranging from the efficiency of the individual grain destruction mechanisms, to more difficult questions concerning how much time an individual grain might spend in cold cores versus the warm ISM. Theoretical astrophysicists have done a great deal of work trying to formulate a comprehensive model to address the efficiency of grain destruction as a function of shock velocity. The general conclusion drawn from such efforts is that an average interstellar grain survives on the order of $10^{8} \mathrm{yr}$ before being destroyed (e.g., Jones et al. 1994, 1996).

\subsection{Modelling vs. observations}

Observations of the elemental depletions in the ISM as a function of the cloud velocity and density along a given line of sight imply that strong shock waves in the low density ISM destroy dust (Routly \& Spitzer 1952; Seab \& Shull 1983). It was not until the detailed studies by Barlow \& Silk (1977), Draine \& Salpeter (1979a,b) and Cowie (1978) that a clear link was made between the enhanced gas phase abundances and the destruction of grains in low velocity shocks. Cowie (1978) shows that for clouds with velocities as low as $40-50 \mathrm{~km} \mathrm{~s}^{-1}$ the abundance of $\mathrm{Si}$ can be cosmic. (Note that these are observed radial velocities, which give only one component of the actual space velocity.) We also note that, although the cloud velocity in this case is, apparently, too low for "complete" dust destruction (e.g., Jones et al. 1996), the shock that accelerated the cloud of gas and dust would have been faster. The density enhancement across a shock front is about a factor of four and, in the LSR, the post-shock gas would be moving at $\sim 3 / 4$ of the shock speed. The observational results of Cowie (1978) could therefore apply to shocks now moving at approximately $55-65 \mathrm{~km} \mathrm{~s}^{-1}$, assuming that the LSR velocity of the preshock gas is close to zero. However, because the shocks are decelerating, the observed post-shock gas could represent gas and dust originally shocked to velocities perhaps as high as $\sim 200 \mathrm{~km} \mathrm{~s}^{-1}$ when the shock would have been 
radiative. For comparison, Jones et al. (1996) find that shocks of velocity $150-200 \mathrm{~km} \mathrm{~s}^{-1}$ destroy only about $30-50 \%$ of the silicate dust. Given the observational and modelling uncertainties a factor of only two difference here corresponds to a rather good agreement between observation and theory. Other works (e.g., Crinklaw et al. 1994; Savage \& Sembach 1996; Jones 2000) build upon this basis and analyse in depth the elemental depletions as a function of environment.

Welty et al. (2002) used near-UV spectra from the Hubble Space Telescope GHRS to measure the abundances and determine the physical conditions within the ISM in high velocity $\left(-105 \mathrm{~km} \mathrm{~s}^{-1} \lesssim v_{\odot} \lesssim-65 \mathrm{~km} \mathrm{~s}^{-1}\right)$ and intermediate velocity $\left(-60 \mathrm{~km} \mathrm{~s}^{-1} \lesssim v_{\odot} \lesssim-10 \mathrm{~km} \mathrm{~s}^{-1}\right)$ gas toward the star $\zeta$ Ori. They find $\mathrm{Al}, \mathrm{Si}$ and $\mathrm{Fe}$ depletions indicative of about $10 \%$ silicate dust destruction, which is consistent with the latest models for silicate grain destruction (Jones et al. 1994, 1996). However, for carbon they find near-solar gas phase abundances indicating 60-70\% carbon dust destruction. Podio et al. (2006) use optical and infrared diagnostics that can be used to probe the dust erosion in Herbig-Haro jets in the Orion and Vela star forming regions. They find substantial, but incomplete, $\mathrm{Ca}$ - and $\mathrm{Fe}$-bearing dust destruction $(0-70 \%$ and $90 \%$ in the gas for $\mathrm{Ca}$ and $\mathrm{Fe}$, respectively). They also find that carbon is not depleted in these shocks with respect to solar abundances, i.e. carbon dust is essentially completely destroyed. Frisch et al. (1999) and Slavin $\&$ Frisch (2006) studied the nature of the dust in the local interstellar wind and cloud and find that the silicate grain elemental depletions ( $\mathrm{Si}, \mathrm{Mg}$ and $\mathrm{Fe}$ ) are consistent with the local cloud having been shocked to a velocity of the order of $150 \mathrm{~km} \mathrm{~s}^{-1}$. However, Slavin \& Frisch (2006) find that the gas phase carbon abundance in the local cloud is at least solar, indicating the complete destruction of the carbon grains in the local ISM that was shocked by the supernova event that gave rise to the local bubble structure (Frisch et al. 1999).

Thus, observational evidence from shocked regions in the ISM indicates high fractional abundances of gas phase carbon, implying that $60-100 \%$ of the carbon dust has been destroyed. For comparison, in quiescent regions of the diffuse ISM $\sim 40 \%$ of the available carbon is observed to be in the gas (Cardelli et al. 1996). For regions of the ISM shocked to velocities of the order of 50-150 $\mathrm{km} \mathrm{s}^{-1}$ (Welty et al. 2002; Podio et al. 2006; Slavin \& Frisch 2006), it appears that the pre-existing carbon dust, whatever its original form, underwent major destruction. These observational results are in contradiction with the predictions of graphite/amorphous carbon dust shock-processing models (Tielens et al. 1994; Jones et al. 1994, 1996), which predict that $\leq 15 \%$ of the carbon dust will be eroded for shock velocities in the range $50-150 \mathrm{~km} \mathrm{~s}^{-1}$, but in better agreement with the recent determinations by Serra Díaz-Cano \& Jones (2008). Their results for an a-C:H carbon dust model for a shock velocity of $100 \mathrm{~km} \mathrm{~s}^{-1}$ are in rather good agreement with the observations, and in much better agreement than the results using graphite/amorphous carbon as the solid carbon phase (Jones et al. 1994, 1996).

\subsection{Dust lifetimes and the global problem}

The determination of the dust lifetime, i.e., its resistance to shock processing, depends on the dust composition and structure (e.g., Jones et al. 1994, 1996). In general such calculations assumed that the dust is principally composed of amorphous silicates (a-Sil) and graphite/amorphous carbon (a-C). The lifetime for hydrogenated amorphous carbon $(\mathrm{a}-\mathrm{C}: \mathrm{H})$ grains in the ISM, of the order of $2 \times 10^{8} \mathrm{yr}$, is shorter by a factor of about three than the value of $\sim 6 \times 10^{8}$ yr derived by Jones et al. (1996) for a-C grains. For a-Sil dust the lifetime is $\sim 4 \times 10^{8} \mathrm{yr}$ (Jones et al. 1996). The shorter lifetime or high destruction rate for grains has major consequences for the evolution of dust in the ISM, it implies that the average cycling time-scale for dust must be short. We may therefore need to focus our attention on how the structure of the ISM phases, and the cycling of matter between those phases, could perhaps help in explaining this rapid cycling requirement.

An argument against rapid, widespread grain destruction in the ISM has been advanced by Mathis (1996) and O'Donnell \& Mathis (1997). Studies of the interstellar extinction curve along various lines of sight have shown that both the grain size distributions and the overall composition of the grain populations are far from constant. The simplest models that successfully account for such variations in, for example, the shape or relative strength of the $217.5 \mathrm{~nm}$ feature versus the strength of the far UV rise (characteristic signatures of interstellar spectra), or the depth of the silicate absorption band, all require separate, independently variable populations of carbonaceous and silicate/oxide grains. Grain destruction, followed by rapid accretion of refractory (including carbon) atoms onto grain surfaces should quickly result in a homogeneous solid state where an average grain contains both silicate/oxide and carbonaceous matter mixed together. No experimentally-supported mechanism has yet been proposed by those advocating the rapid destruction of grains in the ISM that would maintain separate populations of oxide and carbonaceous grains. However, Draine (2009), and earlier works cited therein, has suggested a combination of chemisputtering (by $\mathrm{H}$ and $\mathrm{O}$ ) and/or photodesorption/photolysis processes that could provide the substrate-dependent selectivity necessary to grow separate carbon and silicate dust materials under ISM conditions. A clear problem with this mechanism is that, in addition to silicate and carbon formation, it would also yield a suite of very stable, metal carbide and silicon carbide materials that are not observed in abundance in the ISM. Thus the suggestion of Draine (2009), while not yet a testable hypothesis, and currently unsupported by experimental evidence, must necessarily remain somewhat speculative.

\subsection{Dust and the nature of the shocked ISM}

Current models for dust processing in shock waves generally make the simplifying assumption that the pre-shock medium of the considered ISM phase (e.g., the warm medium Jones et al. 1994, 1996; or a molecular cloud Guillet et al. 2007, 2009, 2011) is homogeneous (e.g., in terms of density, temperature, etc.) and that the medium remains homogeneous during and after the passage of a shock. However, is such a fundamental assumption really valid given the observational evidence? Here we point out that we wish to consider the effects of "small-scale" inhomogeneities in a given medium (i.e., clumps or clumpiness), which is not to be confused with the large-scale multi-phase model of the ISM. In fact, we consider that any considered phase in the multi-phase model is probably, to some extent, clumpy at some level.

For regions of the ISM shocked locally, e.g., regions of active star formation with proto-stellar outflows, the observed shocked fluids are clumped and exhibit chaotic velocity fields (e.g., Bally et al. 2002). The observed structures are indeed rather complex and it appears that pre-shock cooling instabilities could have been instrumental in forming a spray of denser clumps in the post-shock medium (Bally et al. 2002). 
Simulations of triggered star-formation relevant to the formation of the solar system (e.g., Boss et al. 2010) indicate that shock-cloud encounters can lead to cloud collapse and star formation for shock velocities up to $\sim 70 \mathrm{~km} \mathrm{~s}^{-1}$. A general finding of these simulations is that Rayleigh-Taylor instabilities at shock-cloud boundaries lead to a "shredding" of the cloud into cloudlets too small to collapse under their own gravity but still dense enough that the shock passes around them (e.g., Boss et al. 2010).

Recent work on the processing of PAHs in the ISM shows that they are rapidly destroyed in shocks and in a hot gas (Micelotta et al. 2010a,b). In order to explain the PAH emission observed in starburst outflow regions, such as in M 82, Micelotta et al. $(2010 \mathrm{a}, \mathrm{b})$ propose that this emission must trace denser clouds/clumps of matter entrained in the outflow. The observed emission then comes from the "evaporation" of PAHs into the lower density hot gas where they emit but where they are also rapidly destroyed.

As pointed out by Guillard et al. (2009, 2010), in the case of high velocity shocks between the interacting galaxies in Stephan's Quintet, shocked clouds are subject to RayleighTaylor and Kelvin-Helmholtz instabilities and gas coolingthermal instabilities that lead to cloud fragmentation. Their work indicates that such a shock-triggered formation of denser regions and lower density voids, formed on time-scales typical of the gas cooling time-scale, are likely to be at the origin of the molecular hydrogen and dust observed in the environment of galactic collisions.

Thus, from the observational evidence, it appears that the swept up material in shocked regions, over a large range of size scales, is likely to be highly clumpy. These density inhomogeneities in the pre-shocked and post-shocked media could explain how some fraction of the PAHs and dust could be protected and transported through hostile environments to survive and emit in regions where models predict that their survival time-scales are significantly shorter than their transport time-scales. Current models for dust processing in interstellar shock waves, be they supernova- or stellar jet-generated, explicitly ignore any effects of clumping on small size-scales (e.g., Jones et al. 1994, 1996; Guillet et al. 2007, 2009, 2011).

Therefore, the fraction of the dust that is fully exposed to the effects of a shock in a clumpy medium is probably less than the $100 \%$ that is postulated in most studies that assume completely homogeneous media, although we obviously cannot preclude the possibility of total exposure in some cases. In conclusion, it would seem that there is sufficient observational and theoretical evidence that the assumption, that all of the dust and gas in a cloud are exposed to the full effects of a shock, is not justified. This subject obviously merits a more detailed analysis and more sophisticated modelling.

\section{Experimental constraints}

\subsection{Pre-solar grains}

Pre-solar grains, extracted from meteorites and IDPs, originating from a wide variety of stellar sources including red giants, AGB stars, novae, supernovae and Wolf-Rayet stars have been identified based on their isotopic compositions. All of these grains must have passed through the ISM before being incorporated into the molecular cloud that eventually collapsed to form our sun. They should therefore tell us something about the conditions that they experienced.
Studies of meteorite matrix and IDPs have shown that, at a minimum, the following materials are present at some level in interstellar dust: nanodiamonds (Lewis et al. 1989; Bernatowicz et al. 1990), poorly graphitized carbon (Amari et al. 1990), amorphous carbonaceous materials (Zinner 1988), crystalline and amorphous $\mathrm{Al}_{2} \mathrm{O}_{3}$ and $\mathrm{MgAl}_{2} \mathrm{O}_{4}$ (Huss et al. 1994; Nittler et al. 1997; Zinner et al. 2003; Stroud et al. 2004), and crystalline and amorphous silicates (Bradley 1994; Messenger et al. 2003; Nguyen \& Zinner 2004). Given that a large fraction of the material in the meteorite matrix is of nebular origin (Boynton 1985; Clayton 1985) it is surprising that any presolar grains survived at all. Considering the generally oxidizing nature of the solar nebula, it is remarkable that most of the presolar materials isolated to date are carbonaceous grains that are easily destroyed via exposure to even moderately high temperatures in the presence of oxygen. The carbonaceous grains that do survive nebular and parent-body processing - such as $\mathrm{SiC}$ - show no apparent signs on their surfaces of pitting (e.g., Bernatowicz et al. 2003) due to chemi-sputtering or to chipping and microcratering due to grain-grain collision processes that should be evident if the interstellar medium were as violent as predicted (e.g., Jones et al. 1996).

\subsection{Grain formation in the laboratory}

\subsubsection{Silicate grains}

Amorphous magnesium silicate smokes have been prepared in the laboratory by vapor phase condensation and annealed in vacuum to simulate circumstellar processing. The samples can be monitored by IR spectroscopy as a function of annealing time and temperature (1000-1050 K), focusing on the development of the 10- and $20-\mu \mathrm{m}$ silicate features. The IR spectrum of the initial condensates generally display a broad band at $9.3 \mu \mathrm{m}$. As annealing proceeds, the maximum shifts to longer wavelengths and eventually stabilizes at $9.7 \mu \mathrm{m}$, the value typically reported for silicates in the interstellar medium and in the circumstellar outflows of oxygen-rich stars. This type of processing has been followed in detail by Hallenbeck et al. (1998) and Hallenbeck \& Nuth (1998). Further, they found that the thermal evolution of the amorphous magnesium silicate smokes leads to the appearance of a dual maximum at 9.8 and $11.2 \mu \mathrm{m}$, indicative of crystalline olivine. The dual-maxima $10 \mu \mathrm{m}$ feature is a natural consequence of the thermal evolution of the amorphous condensate and is not a mixture of distinct populations of amorphous and crystalline materials.

In addition, there is a natural pause or stall in the spectral evolution of these samples, midway between the initially chaotic condensate and the more ordered solid. Recent work indicates that this stall phase may represent the nucleation of microcrystals at the outer edge of the amorphous grains (Kamitsuji et al. 2005). Thereafter, individual features sharpen as the sample develops a polycrystalline structure that becomes more ordered with further annealing. For the magnesium silicate smoke samples investigated, roughly 11 days of annealing at a grain temperature of $1000 \mathrm{~K}$ were required for the peak position of the $\mathrm{Si}-\mathrm{O}$ stretch to evolve from $9.3 \mu \mathrm{m}$ to $9.7 \mu \mathrm{m}$. An additional eleven days of thermal annealing $(\approx 22$ days total) were required before the grains reached the stall, and it is estimated that nearly three hundred additional days at $1000 \mathrm{~K}$ would be necessary before the infrared spectrum of the annealing magnesium silicate progresses beyond the stall. The stall spectrum is therefore a practical endpoint in the spectral evolution of most magnesium 
silicate condensates in circumstellar outflows, though virtually all grains in the outflow should evolve to exhibit the $9.7 \mu \mathrm{m}$ silicate stretch. Since grain temperatures range from 300-600 K in the outer shells of typical stellar outflows Hron et al. (1997), any magnesium silicate grain sufficiently annealed to exhibit the stall spectrum should remain at this stage of evolution for the remainder of its lifetime, barring shock-induced destruction or radiation induced amorphization in the ISM. Based on the laboratory results discussed above, grains formed at temperatures in excess of $1067 \mathrm{~K}$ anneal from the initial amorphous condensate to a material that displays a spectrum more evolved than the stall on timescales of minutes. At temperatures of $1000 \mathrm{~K}$ these transitions require in excess of 300 days.

In essence, what these experiments show is that it is possible to replicate the spectra of grains formed in circumstellar outflows in the laboratory and that such analogue materials match the observed spectra of the astronomical silicate quite well implying that the simplest explanation for the properties of silicates in the ISM is that they condensed in dense, circumstellar vapors, not in the low-density ISM. On the contrary, vacuum condensation in the laboratory tends to produce metallic films, which would result in a grey extinction in interstellar grains, and in dense, cold, interstellar environments would tend to produce grains with much broader spectral absorption bands than those observed.

\subsubsection{Carbon grains}

Based on a series of catalytic-type reaction experiments Nuth et al. (2008) discuss an interesting mechanism for the formation of macro-molecluar, carbonaceous materials from $\mathrm{CO}$ and $\mathrm{H}_{2}$ on the surfaces of amorphous iron and magnesium silicates and pure silica smokes. This appears to be a viable process for the formation of organic materials under primitive solar nebula conditions. If this process could also operate in the lower density ISM then it might provide a viable route to hydrogenated amorphous carbon grain re-formation. However, it might be that the accretion process in the ISM begins before the formation of $\mathrm{CO}$ and $\mathrm{H}_{2}$ with the re-accretion of the atomic components $(\mathrm{H}, \mathrm{C}$, $\mathrm{O}$, etc.) onto the residual grain surfaces after having been sputtered into the gas in shocked regions. In this case we then also have to explain the chemically-segregated formation of hydrocarbon and silicate grain phases in a gas composed, predominantly, of the condensible elements $\mathrm{H}, \mathrm{C}, \mathrm{O}, \mathrm{Si}, \mathrm{Mg}$ and $\mathrm{Fe}$.

In a more recent work Nuth \& Johnson (2010) studied the reactions of $\mathrm{CO}$ and $\mathrm{H}_{2}$ on graphite surfaces and showed that this can lead to the formation of carbon nano-tubes at temperatures as low as $873 \mathrm{~K}$. Apparently, graphite surfaces can also be efficient catalysts in the formation of species of astrophysical interest, such as carbon nano-tubes.

\section{Resolving the conundrum?}

The timescale for grains, formed in the outflows of dying stars, to be incorporated into new proto-stellar systems is on the order of $10^{9}$ yr (Dwek \& Scalo 1980; Jones \& Tielens 1994). Given that, on average, grains formed, predominantly, around AGB stars and injected into the ISM, only survive of order $10^{8} \mathrm{yr}$ before being destroyed by a supernova shock wave, the inevitable conclusion is that the vast majority of grains now observed in the ISM must have formed there. Jones et al. (1997) estimated that some $10 \%$ of stardust might never have encountered a fast shock before incorporation into the solar system. However, this only gives an idea of the maximum possible presolar stardust contribution because the major fraction of this dust, along with any presolar dust formed in the ISM, would have been reprocessed in the solar nebula and probably have lost all trace of its stardust origin. Thus, the meteoritic presolar dust content must be very small (probably $\lesssim 1 \%$ ). On this basis, finding presolar materials that originated in many different stellar sources would therefore seem to be a very difficult proposition indeed. The result of taking these models of grain destruction to their natural conclusion is that it is very difficult to explain the spectral properties inferred from the interstellar extinction curve and the presence of the vast diversity of pre-solar grains in meteorites and IDPs. The spectral properties of grain analogs formed in the laboratory under conditions appropriate to the ISM are very different from those indicated by the observations. Alternatively, we are forced to postulate that appropriate amorphous silicate and carbonaceous grains simply reform in the ISM by processes that we do not yet understand and have yet to duplicate in the laboratory.

In determining the dust lifetime astrophysicists adopt an effective number of supernovae, derived from the galactic supernova rate, modified to allow for the fact that they occur in associations and not in isolation. This effective rate is then used to calculate the frequency of destructive shocks and the rate at which these shocks destroy dust. The global dust lifetime is then derived by dividing the mass of dust in our galaxy by the rate of grain destruction. What is thus calculated is the time it would take to destroy all of the dust in the galaxy, something that is never likely to occur. This timescale is then defined as the lifetime of the average grain. Such an approach is clearly inadequate to say much about the detailed distribution and more sophisticated models are now needed. Additionally, we may need to revisit the fundamental question of what we really mean by a dust lifetime and whether this term really has any useful physical meaning in the sense that it is currently employed.

\subsection{Uncertainties re-examined}

An assessment of dust lifetimes in the ISM would not be complete without a look at the uncertainties inherent in the current estimates. The key ingredients in these calculations are: the dust destruction efficiency in a $\mathrm{SN}$-shock of a given velocity, the mass of the ISM affected by a given $\mathrm{SN}$, the effective $\mathrm{SN}$ rate and the rate of mass exchange between the components in a multiphase ISM. We now try and assess the effects of the uncertainties in these key elements of the dust lifetime.

\subsubsection{Dust destruction efficiency per shock, $\epsilon$}

As already discussed, the most recent estimates indicate that the dust destruction efficiency, $\epsilon$, in the warm ISM is $\lesssim 0.5$. For example, the most effective dust-destroying shocks, in terms of the interplay between the mass of the ISM affected and the destructive potential as a function of shock velocity, have velocities $\cong 100 \mathrm{~km} \mathrm{~s}^{-1}$. For a $100 \mathrm{~km} \mathrm{~s}^{-1}$ shock Jones et al. (1994, 1996); Serra Díaz-Cano \& Jones (2008) find $\epsilon \simeq 0.2(\simeq 0.3)$ for silicates (hydrogenated amorphous carbons). A key parameter in determining the degree of dust destruction in a given shock is $b \cong\left(B_{0} / 1 \mu \mathrm{G}\right) / \sqrt{ } n_{0}$, where $B_{0}$ is the magnetic field and $n_{0}$ is the density in the medium ahead of the shock. This parameter determines the compression $\left(\propto v_{\text {shock }} / b\right)$ behind the shock and the maximum attainable grain velocity $\left(v_{\max } \propto v_{\text {shock }}^{3 / 2} / b^{1 / 2}\right.$, e.g., McKee 1989) and hence the degree of dust destruction 
there: for a given pre-shock density, the degree of compression decreases as $b$ increases due to increased magnetic pressure. Jones et al. (1994, 1996); Serra Díaz-Cano \& Jones (2008) adopted $b=6\left(B_{0}=3 \mu \mathrm{G}\right.$ and $\left.n_{0}=0.25 \mathrm{~cm}^{-3}\right)$. In their study McKee et al. (1987) found that increasing $B_{0}$ from 1 to $3 \mu \mathrm{G}$ leads to approximately a halving of the dust destruction (see their Table 1) and Jones et al. (1994) found that increasing the density by a factor of 10 (100) increases the dust destruction by factors of $\approx 1.5(\approx 2$, see their Table 2$)$. Thus, $\epsilon$ is somewhat sensitive to $b$ but, given that the adopted warm ISM parameters appear to be well-constrained observationally, it is likely that the current dust destruction efficiencies for a given shock velocity are rather sound. However, a comparison of the model predictions, for a $100 \mathrm{~km} \mathrm{~s}^{-1}$ shock (Jones et al. 1996; Serra Díaz-Cano \& Jones 2008), with the observations of Welty et al. (2002), indicates an agreement to within only a factor of $\approx 2$. Thus, it seems reasonable to assume that the currently calculated values of $\epsilon$ could, conservatively, be uncertain by about $\pm 50 \%$.

\subsubsection{SNe and the multiphase ISM}

In his review McKee (1989) concludes that, in a three-phase ISM, a supernova of energy $E_{51}\left(10^{51} \mathrm{erg}\right)$ can affect the dust within an ISM mass of $M_{\mathrm{s}}(1) \approx 1300 E_{51} M_{\odot}$. However, there is some uncertainty about how much energy SNe actually inject into the ISM and, as McKee (1989) points out, $E_{51}$ could lie in the range $0.3-1.0$. There is also some indication that the energies required to create the observed HI shells in the Milky Way may be somewhat lower than expected (e.g., Ehlerová \& Palouš 2005; Bureau \& Carignan 2002). We therefore consider that the value of $E_{51}$ could be uncertain by almost $50 \%$ and adopt $E_{51}=0.7 \pm 0.3$ to encompass the observed range.

We next consider the effective $\mathrm{SN}$ rate, $\left(\tau_{\mathrm{SN}}^{\prime}\right)^{-1}$, which allows for the fact that $\mathrm{SN}$ occur in associations, and is generally assumed to have a value of $\sim 0.008$, equivalent to one SN every 125 yr (e.g., McKee 1989; Jones et al. 1996). More recent determinations, based on galaxy observations, find a $\mathrm{SN}$ rate $\tau_{\mathrm{SN}}=$ $121 \pm 37$ yr (i.e., $\tau_{\mathrm{SN}}^{-1} \equiv 0.009 \pm 0.003$ ) for a barred spiral galaxy like the Milky Way (Cappellaro et al. 1999). Assuming the McKee (1989) methodology this would be equivalent to an effective $\mathrm{SN}$ rate $\left(\tau_{\mathrm{SN}}^{\prime}\right)^{-1}=0.36 \tau_{\mathrm{SN}}^{-1} \equiv 0.003 \pm 0.001$. To be somewhat conservative here we will assume $\left(\tau_{\mathrm{SN}}^{\prime}\right)^{-1}=0.008 \pm 0.004$, i.e. an uncertainty of $\pm 50 \%$.

Uncertainties in the rates of mass exchange between the different phases in a multi-phase ISM are also expected to be rather large. However, and without detailed modelling, it is rather difficult to assess the likely impact of the related uncertainties. We nevertheless believe that this is a key aspect of dust evolution in the ISM that warrants a much deeper understanding. Hopefully, the new results now emerging from the Herschel and Planck space missions will help to shed some light into this dark corner.

\subsection{The dust lifetime re-visited}

We now try to quantify some of the above ideas by modifying the standard dust lifetime calculation used by (Jones et al. 1994, 1996) which is strictly based upon the "global" method presented by (Dwek \& Scalo 1980; McKee 1989). We can use this method to determine the equilibrium fraction of an element, $\delta_{\text {eq }}$, locked into refractory grains in the ISM. Following the usual terminology here, we define $M$ as the mass of the refractory dust element and $\delta$ as the mass fraction of the element locked into dust. Further, we adopt $\delta_{\text {in }}$ as the fraction of the element in the dust injected into the ISM from stellar sources and $t_{\text {in }}$ as the element injection timescale from these sources. $t_{\mathrm{SNR}}$ is defined as the timescale for supernova shock waves to destroy all of the dust in the ISM. As per McKee (1989), we set $\alpha \delta$ to be the fraction of dust destroyed during star formation: $\alpha=1$ corresponds to the destruction of only the dust incorporated into the star, $\alpha>1 \mathrm{im}$ plies a net dust destruction near the newly formed star and $\alpha<1$ a net dust formation in the proto-stellar environment. $t_{\mathrm{sf}}$ is the star formation timescale. We then have (following McKee 1989)

$$
\begin{aligned}
& \frac{\mathrm{d} M}{\mathrm{~d} t}=-\frac{M}{t_{\mathrm{sf}}}+\frac{M}{t_{\text {in }}} \\
& \frac{\mathrm{d} M \delta}{\mathrm{d} t}=-\frac{\alpha M \delta}{t_{\mathrm{sf}}}+\frac{M \delta_{\text {in }}}{t_{\text {in }}}-\frac{M \delta}{t_{\mathrm{SNR}}}
\end{aligned}
$$

which can be combined to give an expression for the equilibrium fraction of an element in dust in the ISM,

$$
\delta_{\mathrm{eq}} \approx \frac{\delta_{\text {in }}}{1+\left(t_{\text {in }} / t_{\mathrm{SNR}}\right)+(\alpha-1)\left(t_{\mathrm{in}} / t_{\mathrm{sf}}\right)} .
$$

For illustrative purposes we will assume that star formation has no net effect on the dust (i.e., $\alpha=1$, the effects of this simplifying assumption will be re-examined later) and we then simply have,

$\delta_{\mathrm{eq}} \approx \frac{\delta_{\mathrm{in}}}{1+\left(t_{\mathrm{in}} / t_{\mathrm{SNR}}\right)}$.

If we now adopt $\delta_{\text {in }}=0.9$ (Jones \& Tielens 1994) and the typical timescales $t_{\text {in }} \sim 3 \times 10^{9} \mathrm{yr}$ (Dwek \& Scalo 1980; Jones $\&$ Tielens 1994), $t_{\mathrm{SNR}} \sim 4 \times 10^{8} \mathrm{yr}$ for $\mathrm{Si}, \mathrm{Mg}$ and $\mathrm{Fe}$ in silicates (Jones et al. 1996) and $t_{\mathrm{SNR}} \sim 2 \times 10^{8} \mathrm{yr}$ for $\mathrm{C}$ in hydrogenated amorphous carbon grains (Serra Díaz-Cano \& Jones 2008) then we find that the maximum fractions of the elements in stardust are $\delta_{\text {eq }}=0.11$ for silicates and $\delta_{\text {eq }}=0.06$ for hydrogenated amorphous carbons. Note that these results are slightly different from the earlier estimates (Jones et al. 1994), due to the more recent evaluations of the degree of carbon dust processing in shocks (Jones et al. 1996; Serra Díaz-Cano \& Jones 2008). These results therefore imply that $90-95 \%$ of the dust mass in the ISM must have been completely re-processed and therefore re-formed in the ISM, as discussed in detail in Sect. 4.

It therefore seems that the fraction of stardust silicates that is preserved "intact" should be rather small because we see basically no crystalline silicates in the ISM. However, the silicate mass could be preserved but the crystalline silicates be rendered amorphous. The fraction of crystalline silicate in stardust formed around evolved stars is probably of the order of $10 \%$, i.e., $\delta_{\text {in }}=$ 0.1 and, following on from above, we see that $\delta_{\text {eq }}=0.01$, which appears to be consistent with the observations showing a lack of crystalline silicates in the ISM (Kemper et al. 2004). However, this is not strictly valid because this preserved "crystalline" fraction will in fact have been rendered amorphous by the same processes (e.g., ion irradiation in shocks) that led to the dust destruction (e.g., Demyk et al. 2001). Given, in particular the apparent difficulty of re-forming silicate grains in the ISM, the results of these simple calculations of $\delta_{\text {eq }}$ are clearly in conflict with the observations of the dust in the ISM and the analysis of the presolar dust grains extracted from meteorites and IDPs that we discussed earlier in Sect. 5.

The basic problem with this approach to the dust lifetime calculation is that it implicitly assumes the (unlikely) "instantaneous" and "global" processing of dust. However, it is clear that dust processing must occur on local scales. In Sect. 4 we showed 
that on local scales the dust destruction in individual processing events (e.g., shock waves) is consistent with our current models. The problem therefore arises when we apply these "localised" results on a Galactic scale.

We now re-examine the lifetime calculation taking into account the uncertainties in the key parameters $\epsilon, \tau_{\mathrm{SN}}$ and $M_{\mathrm{S}}(1)$. Following McKee (1989) and Jones et al. (1994) $t_{\text {SNR }}$ can be calculated as follows:

$t_{\mathrm{SNR}}=\frac{M_{\mathrm{ISM}} \times \tau_{\mathrm{SN}}}{\int \epsilon\left(v_{\mathrm{S}}\right) \mathrm{d} M_{\mathrm{S}}\left(v_{\mathrm{S}}\right)} \mathrm{yr}$,

where $M_{\text {ISM }}=4.5 \times 10^{9} M_{\odot}$ is the mass of the ISM (gas and dust), the effective $\mathrm{SN}$ rate $\tau_{\mathrm{SN}}=125 \mathrm{yr}, M_{\mathrm{S}}$ is the mass of the ISM shocked to a velocity of at least $v_{\mathrm{s}}$, and following Jones et al. (1994) we have $M_{\mathrm{s}}\left(v_{\mathrm{s}}\right)=2914 M_{\odot} v_{\mathrm{s} 7}^{2}$ and

$t_{\mathrm{SNR}}=\frac{4.5 \times 10^{9} M_{\odot} \times \tau_{\mathrm{SN}}}{2 \times 2914 \times \int\left(\epsilon\left(v_{\mathrm{s} 7}\right) / v_{\mathrm{s} 7}^{3}\right) \mathrm{d} v_{\mathrm{s} 7}} \mathrm{yr}$,

where $\epsilon\left(v_{\mathrm{s} 7}\right)$ is the typical dust destruction efficiency as a function of the shock velocity $v_{\mathrm{s}}$, which is expressed in units of $10^{7} \mathrm{~cm} \mathrm{~s}^{-1}$ (i.e., $100 \mathrm{~km} \mathrm{~s}^{-1}$ ). Using the latest dust destruction results for silicates (Jones et al. 1996) and hydrogenated amorphous carbon or a-C:H (Serra Díaz-Cano \& Jones 2008), $\epsilon\left(v_{\mathrm{s} 7}\right)$ can be fitted with the simple analytical form $\epsilon\left(v_{\mathrm{s} 7}\right)=$ $\left(2 v_{\mathrm{s} 7}-1\right) / n$ (where $n=6$ for silicate dust and 3 for a-C:H dust) where we take only the positive values. This simple fit leads to a good agreement with the previously-derived values for $t_{\mathrm{SNR}}$ (Jones et al. 1996; Serra Díaz-Cano \& Jones 2008) and also reproduces the detailed destruction as a function of the shock velocity to within less than a factor of two. Adopting this simple analytical form the integral in Eq. (6) is evaluated as $1.1 / n$ and we then have,

$t_{\mathrm{SNR}}=\frac{4.5 \times 10^{9} M_{\odot} \times \tau_{\mathrm{SN}}}{2 \times 2914 \times(1.1 / n)} \mathrm{yr}$.

Substituting for $\tau_{\mathrm{SN}}$ and adding the uncertainties estimated in the previous section we have

$t_{\mathrm{SNR}}=\frac{n \times\left[(4.5 \pm 2.2) \times 10^{9}\right] \times(125 \pm 62)}{2 \times(2914 \pm 870) \times(1.1 \pm 0.6)} \mathrm{yr}$

where we have also assumed an uncertainty of $\pm 50 \%$ for the mass of the ISM in the Milky Way. Combining the uncertainties in quadrature we find that the overall uncertainty is of the order of $90 \%$, i.e.,

$t_{\mathrm{SNR}} \approx n \times(8.8 \pm 7.9) \times 10^{7} \mathrm{yr}$.

This estimation yields values of $\sim(0.3-10.0) \times 10^{8} \mathrm{yr}$ and $\sim(0.2-5.1) \times 10^{8} \mathrm{yr}$ for the silicate and a-C:H grain "global" lifetimes, respectively. If, as discussed in the previous section, the magnetic field strength in the warm ISM has been underestimated, then $\epsilon$ will have been somewhat overestimated. Taking into account the combination of the likely uncertainties in the dust lifetime determination we can see that the silicate dust lifetime is not entirely incompatible with the typical dust injection time-scale of $t_{\text {in }} \sim 2 \pm 1 \times 10^{9} \mathrm{yr}$ (e.g., Dwek \& Scalo 1980; McKee 1989; Jones \& Tielens 1994). Thus, the time-scale discrepancies for silicate dust look less worrying but are probably still significant enough for carbon dust that we have to worry about its (re-)formation in the ISM. The equivalent equilibrium fraction of an element in stardust in the ISM is now given by

$\delta_{\text {eq }} \approx \delta_{\text {in }}\left(1+\frac{t_{\text {in }}}{t_{\text {SNR }}}\right)^{-1}=\delta_{\text {in }}\left(1+\frac{(2 \pm 1) \times 10^{9}}{n \times(8.8 \pm 7.9) \times 10^{7}}\right)^{-1}$, where the uncertainties on $\delta_{\text {eq }}$ are of order $100 \%$ !

Note that in the above we have not taken into account any uncertainties associated with mass transfer between the various ISM phases. From the observational results discussed in Sect. 4.3 it appears that in shocked environments matter is entrained into shock-formed or shock-enhanced clumps, as observed in stellar and galactic winds expanding into inhomogenous media (e.g., Chevalier \& Clegg 1985; Suchkov et al. 1994; Strickland \& Stevens 2000; Marcolini et al. 2005, see also the discussion in Sect. 4.3). It is therefore possible that the dense clumps could protect the interior gas and dust and shield them from the destructive effects of the shock and the hot post-shock gas. (The dense clumps will, given the right conditions and sufficient time, tend to "evaporate".) We do not consider this possibility further here because it appears that any "entrainment" would have to be very efficient at protecting matter, maybe unrealistically so. Thus, the issues related to the structure of the ISM and the mass transfer between the various phases clearly need to be fully investigated with more sophisticated models.

\subsection{The effects of stars and star formation re-examined}

In the previous section we made the simplifying assumption that star formation has no net effect on the dust (i.e., $\alpha=1$ ). A closer examination of this assumption, in the light of the use of this parameter in determining the equilibrium fraction of stardust in the ISM (see Eq. (3)), reveals that it could be of critical importance. Indeed Burke \& Silk (1976) pointed out that proto-stellar discs could be important sites of silicate grain formation.

In general, the star formation timescale, $t_{\text {sf }}$, is defined as $M / \dot{M}$ and, given a Milky Way star formation rate of $\sim 3 M_{\odot} \mathrm{yr}^{-1}$ and an interstellar gas mass of $\sim 4.5 \times 10^{9} M_{\odot}$, implies $t_{\mathrm{sf}} \sim 1.5 \times 10^{9} \mathrm{yr}$ and that the ratio $t_{\mathrm{in}} / t_{\mathrm{sf}}$ is of order 2 . Thus, only a rather large net formation (or loss) of dust during star formation $(\alpha<0.5)$ could have any major impact on dust cycling in the ISM.

Given that the silicate materials in the ISM already contain the majority of the condensable oxide and silicate forming elements ( $\mathrm{Si}, \mathrm{Mg}$ and $\mathrm{Fe}$ ) in the quiescent ISM, processing during star formation could have little effect on the silicate stardust content. However, for carbonaceous materials in the ISM, where only about half of the carbon is to be found in a solid form, the effect could be important if the star formation process is a net carbon dust source. This would be possible if a major fraction $(>0.5)$ of the carbon is incorporated, or re-incorporated, into dust and subsequently ejected into the ISM as intact grains from the star formation site. This could occur in molecular clouds by the direct accretion of carbonaceous mantles onto pre-existing grains, or the accretion of ice mantles in dense regions and their radiative re-processing to more refractory materials with the onset of star formation (see Sect. 3.2). In this case it would seem that the star formation process in molecular clouds could be a source of carbon dust re-processing and re-formation.

Evidence for a net dust formation and injection from stellar regions may also come from the observation of the $\beta$-meteoroids that are ejected from the solar system by solar radiation pressure (Zook \& Berg 1975; Grün et al. 1992). In addition, the largescale circulation pattern in the solar nebula - and presumably in all protostars - carries materials processed in the innermost nebular regions out to at least 40-50 AU, the region where Kuiper Belt comets form. This large-scale cycling has been confirmed by the results of the STARDUST mission which recovered chondrule- and CAI-like fragments from comet Wild 2 (a Kuiper Belt Comet) that could never have been 
closer to the sun than $40 \mathrm{AU}$, though it must certainly have formed much farther away (Zolensky et al. 2006, see also the 2009, M\&PS STARDUST Special Issue, Vol. 44, No. 10). The Fischer-Tropsch type synthesis of carbonaceous materials is a process that appears to work within the inner nebula. The products of this reaction could clearly also be carried from the inner nebula and out to the Kuiper Belt and possibly beyond (e.g., Nuth \& Johnson 2010). From these mass transfer circulation patterns, and the effects of (proto-)stellar jets, both carbon dust and crystalline and amorphous silicates, could have been formed or processed in the inner nebulae and transported out to the edge of the solar system, possibly to be lost via the processes that eventually clear out these nebular regions, e.g., T Tauri winds. For carbon, this could lead to a net increase in the mass of dust in the ISM while for silicates this could increase the mass fraction of crystalline materials in the local dust population.

Interestingly, there appears to be a rather significant flux of (nano-)particles, originating in the inner solar system close to the Sun; their detection and possible origins have been extensively discussed by Le Sergeant \& Lamy (1978) and Meyer-Vernet et al. (2009).

It is possible that such processes, especially for carbonaceous matter, could seed the local ISM with (nano-)particles that can then act as accretion centres for gas phase refractory atoms and, thus, lead to dust mass re-formation. This could be particularly important in regions such as the Local Bubble where practically all of the available carbon has been eroded into the gas by the effects of supernova-generated shocks (e.g., Slavin \& Frisch 2006). For silicate materials their erosion in the Local Bubble is significantly less $(\sim 10-20 \%$ destruction) than for carbon-bearing dust $(\sim 100 \%$ destruction $)$ and so the need for silicate/oxide dust (re-)formation, or re-accretion, locally is relaxed.

\section{Concluding remarks}

Current methods used to determine the "lifetime" of dust in the ISM $\left(\approx 10^{8} \mathrm{yr}\right)$, that adopt a "global" or galactic-scale approach, present certain difficulties with respect to a number of observable quantities, notably:

- Laboratory analyses of pre-solar grains, present in meteorites and interplanetary dust particles at about the one percent level, that indicate that not all dust is destroyed/re-processed in the ISM but that some, non-negligble, fraction of stardust is transported through the ISM intact, from many tens of different stardust sources, and preserves a memory of its origin.

- The apparent, fast re-cycling of carbonaceous matter in the ISM, which seems counter to the presence of rather "volatile", oxidisable, carbon-rich pre-solar grains.

- The extreme difficulty in forming, from atomic, ionic or molecular constituents, any sort of silicate/oxide phase in the low density and low pressure ISM with spectral properties even remotely similar to those observed.

- Efficient dust destruction implies the efficient re-formation of separate populations of carbonaceous and silicate/oxide grains in the tenuous ISM from a gas of approximately "cosmic" composition; i.e., from the (atomic) products of dust erosion in shock waves.

\section{Laboratory and astrophysical observations imply that:}

- Dust consists, primarily, of two compositionally-distinct phases, namely carbonaceous and silicate solids.
- The carbonaceous and silicate dust populations must, predominantly, be in separate grain populations, as exemplified by the lack of a polarisation signature in the $3.4 \mu \mathrm{m}$ carbonaceous band (cf., the observed polarisation in the $9.7 \mu \mathrm{m}$ silicate band).

- The majority of the condensible, dust-forming elements are to be found in dust in the ISM outside of shocked regions.

- Dust is locally destroyed in the ISM in interstellar and circumstellar shocks.

- Carbon dust is more readily destroyed than silicate/oxide dust.

Some remaining conundrums:

- The, apparently, efficient destruction of carbonaceous dust in shock waves implies that it must be rapidly re-formed in the ISM, most likely by re-accretion onto surviving carbonaceous and silicate grain surfaces. Yet polarisation observations indicate that re-formed carbonaceous matter must occur as a dust component distinct from the amorphous silicate phase.

- Do cosmic rays destroy/amorphize a significant quantity of dust in the ISM on short time-scales? The presence of "pristine" pre-solar grains that existed in the galactic ISM for, probably, hundreds of millions of years before their incorporation into the solar system might seem to imply that cosmic rays are not an important dust processing agent.

- Can grains that are inside denser clumps that interact with supernova shocks be shielded from destruction to some extent as the shock wave "shreds" the dense cloud?

It appears that the current estimates of "global" dust lifetimes could be uncertain by factors large enough to call into question their usefulness.

Acknowledgements. We wish to thank our numerous colleagues and friends for interesting and stimulating discussions on dust evolution in the ISM. This research was made possible through the key financial support of the Agence National de la Recherche (ANR) through the program Cold Dust (ANR-07BLAN-0364-01).

\section{References}

Adamson, A. J., Whittet, D. C. B., \& Duley, W. W. 1990, MNRAS, 243, 400 Adamson, A. J., Whittet, D. C. B., Chrysostomou, A., et al. 1999, ApJ, 512, 224 Amari, S., Anders, A., Virag, A., \& Zinner, E. 1990, Nature, 345, 238 Bally, J., Heathcote, S., Reipurth, B., et al. 2002, ApJ, 123, 2627

Barlow, M. J., \& Silk, J. 1977, ApJ, 211, L83

Bernatowicz, T. J., \& Cowisk, R. 1997, in Astrophysical implications of the laboratory study of presolar materials, AIP Conf. Proc., 402, 451

Bernatowicz, T. J., Gibbons, P. C., \& Lewis, R. S. 1990, ApJ, 359, 246

Bernatowicz, T. J., Messenger, S., Pravdivtseva, O., Swan, P., \& Walker, R. M. 2003, Geochim. Cosmochim. Acta, 67, 4679

Bernatowicz, T. J., Akande, O. W., Croat, T. K., \& Cowsik, R. 2005, ApJ, 631, 988

Bernstein, M. P., Sandford, S. A., Allamandola, L. J., Gillette, J. S. B., Clemett, S. J., Zare, R. N. 1999, Science, 283, 1135

Bernstein, M. P., Dworkin, J. P., Sandford, S. A., Allamandola, L. J. 2001, M\&PS, 36, 351

Bernstein, M. P., Elsila, J. E., Dworkin, J. P., et al. 2002, ApJ, 576, 1115

Boss, A. P., Keiser, S. A., Ipatov, S., Myhill, E., \& Vanhala, H. T. 2010, ApJ, 708,1268

Boynton, W. V. 1985, in Protostars and planets II (University of Arizona Press), 772

Bradley, J. P. 1994, Science, 265, 925

Bringa, E. M., Kucheyev, S. O., Loeffler, M. J., et al. 2007, ApJ, 662, 372

Bureau, M., \& Carignan, C. 2002, AJ, 123, 1316

Burke, J. R., \& Silk, J. 1976, ApJ, 210, 341

Butchart, I., McFadzean, A. D., Whittet, D. C. B., et al. 1986, A\&A, 154, L5

Cappellaro, E., Evans, R., \& Turatto, M. 1999, A\&A, 351, 459

Cardelli, J., Meyer, D. M., Jura, M., \& Savage, B. D. 1996, ApJ, 467, 334 
Chevalier, R. A. \& Clegg, A. W. 1985, Nature, 317, 44

Chiar, J. E., Pendleton, Y. J., Geballe, T. R., \& Tielens, A. G. G. M. 1998, ApJ, 507,281

Chiar, J. E., Adamson, A., Whittet, D. C. B., et al. 2006, ApJ, 651, 268

Clayton, R. N., Mayeda, T. K., \& Molini-Velsko, C. A. 1985, in Protostars and planets II (University of Arizona Press), 755

Clayton, D. D., Liu, W., \& Dalgarno, A. 1999, Science, 283, 1290

Cohen, M., Allamandola, L., Tielens, A. G. G. M., et al. 1986, ApJ, 302, 737

Cowie, L. L. 1978, ApJ, 225, 887

Crinklaw, G., Federman, S. R., \& Joseph, C. L. 1994, ApJ, 424, 748

Dartois, E., Muñoz-Caro, G. M., Deboffle, D., \& d'Hendecourt, L. 2004, A\&A, 423, L33

Davoisne, C., Leroux, H., d'Hendecourt, L., Jones, A. P., \& Deboffle, D. 2006, A\&A, 448, L1

Day, K. L. 1974, ApJ, 192, L15

Day, K. L. 1977, MNRAS, 178, 49

Demyk, K., Carrez, Ph., Leroux, H., et al. 2001, A\&A, 368, L38

Dorschner, J., Begemann, B., Henning, T., Jaeger, C., \& Mutschke, H. 1995, A\&A, 300, 503

Draine, B. T. 2009, ASP Conf. Ser., 414, 453

Draine, B. T., \& Salpeter, E. E. 1979a, ApJ, 231, 77

Draine, B. T., \& Salpeter, E. E. 1979b, ApJ, 231, 438

Duley, W. W. 1985, MNRAS, 215, 259

Duley, W. W. 2000, ApJ, 528, 841

Duley, W. W., Jones, A. P., \& Williams, D. A. 1989, MNRAS, 236, 709

Dwek, E., \& Scalo, J. M. 1980, ApJ, 239, 193

Dworkin, J. P., Deamer, D. W., Sandford, S. A., \& Allamandola, L. J. 2001, Proceedings of the National Academy of Science, 98, 815

Ehlerová, S., \& Palouš, J. 2005, A\&A, 437, 101

Evans, A., Tyne, V. H., Smith, O., et al. 2005, MNRAS, 360, 1483

Fleischer, A. J., Gauger, A., \& Sedlmayr, E. 1991, A\&A, 242, L1

Fleischer, A. J., Gauger, A., \& Sedlmayr, E. 1992, A\&A, 266, 321

Fleischer, A. J., Gauger, A., \& Sedlmayr, E. 1995, A\&A, 297, 543

Frisch, P. C., Dorschner, J. M., Geiss, J., et al. 1999, ApJ, 525, 492

Goto, M., Gaessler, W., Hayano, Y., et al. 2003, ApJ, 589, 419

Greenberg, J. M. 1983, in Cosmochemistry and the Origin of Life, ed. C. Ponnemperuma (Dordrecht: D. Reidel Press), 71

Greenberg, J. M. 1989, Adv. Space Res., 9, 13

Greenberg, J. M., Shalabiea, O. M., Mendoza-Gómez, C. X., Schutte, W., \& Gerakines, P. A. 1995, Adv. Space Res., 16, 9

Gray, M. D., \& Edmunds, M. G. 2004, MNRAS, 349, 491

Grün, E., Zook, H. A., Baguhl, M., et al. 1992, Science, 257, 1550

Guilard, P., Boulanger, F., Pineau des Forêts, G., \& Appleton, P. N. 2009, A\&A, 502, 515

Guilard, P., Boulanger, F., Cluver, M. E., et al. 2010, A\&A, 518, A59

Guillet, V., Pineau des Forêts, G., \& Jones, A. P. 2007, A\&A, 476, 263

Guillet, V., Jones, A. P., \& Pineau des Forêts, G. 2009, A\&A, 497, 145

Guillet, V., Pineau des Forêts, G., \& Jones, A. P. 2011, A\&A, 527, A123

Hallenbeck, S. L., \& Nuth, J. A. 1998, Ap\&SS, 255, 427

Hallenbeck, S. L., Nuth, J. A., \& Daukantas, P. L. 1998, Icarus, 131, 198

Hallenbeck, S. L., Nuth, J. A., \& Nelson, R. N. 2000, ApJ, 535, 247

Hecht, J. H. 1991, ApJ, 367, 635

Henning, T., \& Mutschke, H. 1997, A\&A, 327, 743

Henning, Th., Begemann, B., Mutschke, H., \& Dorschner, J. 1995, A\&AS, 112, 143

Hron, J., Loidl, R., Hofner, S., et al. 1997, A\&A, 335, L69

Hudson, R. L., \& Moore, M. H. 2004, Icarus, 172, 466

Huss, G. R., Fahey, A. J., Gallino, R., \& Wasserburg, G. J. 1994, ApJ, 430L, 81

Jäger, C., Fabian, D., Schrempel, F., et al. 2003, A\&A, 401, 57

Jenkins, E. B. 2009, ApJ, 700, 1299

Jeong, K. S., Winters, J. M., Le Bertre, T., \& Sedlmayr, E. 2003, A\&A, 407, 191

Jones, A. P. 1990, MNRAS, 247, 305

Jones, A. P. 2000, JGR-Space Phys., 105, 10257

Jones, A. P. 2005, Astrochemistry: Recent Successes and Current Challenges, poster session 3: poster 34, IAU Symp., 231

(http: //cdsads.u-strasbg. fr/abs/2005IAUS . 235P.124 J)

Jones, A. P. 2010, in Cosmic Dust - Near and Far, ed. Th. Henning, E. Gruen, \& J. Steinacker, in press

Jones, A. P., \& d'Hendecourt, L. D. 2000, A\&A, 355, 1191

Jones, A. P., \& Tielens, A. G. G. M. 1994, in The Cold Universe, Proceedings of the XXVIIIth rencontre de Moriond, XIIIth Moriond Astrophysics Meeting, ed. T. Montmerle, C. J. Lada, I. F. Mirabel, \& J. Tran Thanh Van (Gif-surYvette: Éditions Frontières), 35

Jones, A. P., Duley, W. W., \& Wiliams, D. A. 1990, QJRAS, 31, 567

Jones, A. P., Tielens, A. G. G. M., Hollenbach, D. J., \& McKee, C. F. 1994, ApJ, 433, 797

Jones, A. P., Tielens, A. G. G. M., \& Hollenbach, D. J. 1996, ApJ, 469, 740
Jones, A. P., Tielens, A. G. G. M., Hollenbach, D. J., \& McKee, C. F. 1997, in The Astrophysical Implications of the Laboratory Study of Presolar Materials, ed. T. J. Bernatowicz, \& E. Zinner, AIP: Conf. Proc., 402, 595

Jones, A. P., d'Hendecourt, L. B., Sheu, S.-Y., et al. 2004, A\&A, 416, 235

Kamitsuji, K., Sato, T., Suzuki, H., \& Kaito, C. 2005, A\&A, 436, 165

Keller, L. P., \& Messenger, S. 2004, in Lunar and Planetary Institute Conf. Abstracts, ed. S. Mackwell, \& E. Stansbery, Lunar and Planetary Inst. Technical Report, 35, 1985

Kemper, F., Vriend, W. J., \& Tielens, A. G. G. M. 2004, ApJ, 609, 826

Kozasa, T., \& Hasegawa, H. 1987, Prog. Theor. Phys., 77, 1402

Krätschmer, W., \& Huffman, D. R. 1979, Ap\&SS, 61, 195

Le Sergeant, L. B., \& Lamy, P. L. 1978, Nature, 276, 800

Leger, A., \& Puget, J.-L. 1984, A\&A, 137, L5

Lewis, R. S., Anders, E., \& Draine, B. T. 1989, Nature, 339, 117

Li, A., \& Draine, B. T. 2001, ApJ, 550, 213

Marcolini, A., Strickland, D. K., D’Ercole, A., Heckman, T. M., \& Hoopes, C. G. 2005, MNRAS, 362, 626

Mason, R. E., Wright, G. S., Adamson, A., \& Pendleton, Y. 2007, ApJ, 656, 798

Mathis, J. S. 1996, ApJ, 472, 643

Mathis, J. S., Rumpl, W., \& Nordsieck, K. H. 1977, ApJ, 217, 425

Mauron, N., \& Huggins, P. J. 1999, A\&A, 359, 707

Mennella, V., Colangeli, L., Bussoletti, E., Palumbo, P., \& Rotundi, A. 1998, ApJ, 507, L177

McKee, C. F. 1989, in Interstellar Dust, International Astronomical Union, ed. L. J. Allamandola, \& A. G. G. M. Tielens (Dordrecht: Kluwer Academic Publishers), Symp., 135, 431

McKee, C. F., Hollenbach, D. J., Seab, C. G., \& Tielens, A. G. G. M. 1987, ApJ, 318,674

Messenger, S., \& Walker, R. M. 1996, in Physics, chemistry and dynamics of interplanetary dust, ed. Bo A. S. Gustafson, \& M. S. Hanner, ASP Conf. Ser., San Francisco, 104, 287

Messenger, S., Keller, L. P., Stadermann, F. J., et al. 2003, Science, 300, 105

Meyer, B. S., \& Zinner, E. K. 2006, in Meteoritics in the Early Solar System II, ed. D. S. Lauretta, \& H. Y. McSween, Jr. (Tucson: AZ. Arizona Press), 69

Meyer-Vernet, N., Maksimovic, M., Czechowski, A., et al. 2009, Solar Phys., 256, 463

Micelotta, E., Jones, A. P., \& Tielens, A. G. G. M. 2010a, A\&A, 510, A36

Micelotta, E., Jones, A. P., \& Tielens, A. G. G. M. 2010b, A\&A, 510, A37

Michael, B. P., Lilleleht, L. U., \& Nuth, J. A. 2003, ApJ, 590, 579

Mutschke, H., Dorschner, J., Henning, T., Jager, C., \& Ott, U. 1995, ApJ, 454, 157

Nittler, L. R., Alexander, C. M. O’d., Gao, X., Walker, R. M., \& Zinner, E. 1997, ApJ, 483, 475

Nuth, J. A. 1996, in International School of Space Chemistry: The Cosmic Dust Connection, ed. J. M. Greenberg, \& V. Pirronello (Dordrecht: Kluwer), NATO, Adv. Study Inst., 205

Nuth, J. A., \& Moore, M. H. 1989, in Lunar and Planetary Science Conference (Cambridge University Press/Lunar and Planetary Institute), 565

Nuth, J. A. III, \& Hecht, J. H. 1990, Ap\&SS, 163, 79

Nuth, J. A. III, \& Johnson, N. M. 2010, ApJ, 710, L98

Nuth, J. A. III, Hallenbeck, S. L., \& Rietmeijer, F. J. M. 1998, Earth, Moon \& Planets, 80, 73

Nuth, J. A., Hallenbeck, S. L., \& Rietmeijer, F. J. M. 2000, JGR, 105, 10387

Nuth, J. A. III, Rietmeijer, F. J. M., \& Hill, H. G. M. 2002, M\&PS, 37, 1579

Nuth III, J. A., Kimura, Y., Lucas, C., et al. 2008, ApJ, 673, L225

Nguyen, A. N., \& Zinner, E. 2004, Science, 303, 1496

O’Donnell, J. E., \& Mathis, J. S. 1997, ApJ, 479, 806

Pendleton, Y. J., Sandford, S. A., Allamandola, L. J., Tielens, A. G. G. M., \& Sellgren, K. 1994, ApJ, 437, 683

Petrie, S., Stranger, R., \& Duley, W. W. 2003, ApJ, 594, 869

Pety, J., Teyssier, D., Fossé, D., et al. 2005, A\&A, 435, 885

Pino, T., Dartois, E., Cao, A.-T., et al. 2008, A\&A, 490, 665

Podio, L., Bacciotti, F., Nisini, B., et al. 2006, A\&A, 456, 189

Routly, P. M., \& Spitzer, L. 1952, ApJ, 115, 227

Sakata, A., Wada, S., Okutsu, Y., Shintani, H., \& Nakada, Y. 1983, Nature, 301, 493

Sandford, S. A., Allamandola, L. J., Tielens, A. G. G. M., et al. 1991, ApJ, 371, 607

Sandford, S. A., Allamandola, L. A., \& Bernstein, M. P. 1997, in From Stardust to Planetesimals, ed. Y. J. Pendleton, \& A. G. G. M. Tielens, ASP Conf. Ser., 122,201

Savage, B. D., \& Sembach, K. R. 1996, ARA\&A, 34, 279

Schutte, W. A. 1996, in Molecules in astrophysics: probes \& processes, ed. E. F. van Dishoeck, IAU symp., 178, 331

Seab, C. G., \& Shull, J. M. 1983, ApJ, 275, 652

Serra Díaz-Cano, L., \& Jones, A. P. 2008, A\&A, 492, 127

Sheu, S.-Y., Lee, I.-P., Lee, Y. T., \& Chang, H.-C. 2002, ApJ, 581, L55 
A\&A 530, A44 (2011)

Shore, S. N.. \& Gehrz, R. D. 2004, A\&A, 417, 695

Slavin, J. D., \& Frisch, P. C. 2006, ApJ, 651, L37

Sorrell, W. H. 1989, MNRAS, 241, 89

Sorrell, W. H. 1990, MNRAS, 243, 570

Sorrell, W. H. 1991, MNRAS, 248, 439

Strickland, D. K., \& Stevens, I. R. 2000, MNRAS, 314, 511

Stroud, R. M., Nittler, L. R., \& Alexander, C. M. O'D. 2004, Science, 305, 1455

Suchkov, A. A., Balsara, D. S., Heckman, T. M., \& Leitherer, C. 1994, ApJ, 430, 511

Tielens, A. G. G. M., McKee, C. F., Seab, G. C., \& Hollenbach, D. J. 1994, ApJ, 431,321
Waters, L. B. F. M., Molster, F. J., de Jong, T., et al. 1996, A\&A, 315, 361

Waelkens, C., Waters, L. B. F. M., de Graauw, M. S., et al. 1996, A\&A, 315, 245

Welty, D. E., Jenkins, E. B., Rayomnd, J. C., \& Mallouris, C. 2002, ApJ, 579, 304

Whittet, D. C. B. 2009, ApJ, 710, 1009

Zinner, E., Ming, T., \& Anders, E. 1988, Nature, 331, 548

Zinner, E., Amari, S., Guinness, R., et al. 2003, Geochim. Cosmochim. Acta, 67, 5083

Zolensky, M. E., Zega, T. J., Yano, H., et al. 2006, Science, 314, 1735

Zook, H. A., \& Berg, O. E. 1975, Planet. Space Sci., 23, 183 